\title{
The Worst-Case Conditions of Endosseous Dental Implants Vary in Implant Diameter and Length: A Finite Element Analysis
}

\author{
Jinyang Zhang, Maisheng Li, Xiao Zhang, and Xianshuai Chen
}

\begin{abstract}
For the dental implant which is available in various dimensions and/or configurations, the dynamic testing should be performed under "worst-case" condition. Thus, a finite element analysis was applied to investigate the effect of implant diameter and length on the stress distribution of dental implant and find out the worst-case condition.

In this paper, six models were computer-simulated by varying the implant diameters $(3.5,4.3$ and $5.0 \mathrm{~mm})$ and lengths $(8$ and $15 \mathrm{~mm})$. The models were used to investigate the influence of length factor: (1) implants with a diameter of $3.5 \mathrm{~mm}$ and lengths of $8 \mathrm{~mm}$ and $15 \mathrm{~mm}$; (2) implant diameters of $4.3 \mathrm{~mm}$ and lengths of $8 \mathrm{~mm}$ and $15 \mathrm{~mm}$; (3) implant diameters of $5.0 \mathrm{~mm}$ and lengths of $8 \mathrm{~mm}$ and $15 \mathrm{~mm}$. The influence of implant diameters was modeled using implant with a length of $8 \mathrm{~mm}$ and diameter of $3.5,4.3$ and $5.0 \mathrm{~mm}$, length of $15 \mathrm{~mm}$ and diameter of $3.5,4.3$ and $5.0 \mathrm{~mm}$. One vertical load $(200 N)$ was applied on the horizontal plane of the crown. A pre-torque of $30 \mathrm{~N} \cdot \mathrm{cm}$ on the central screw was loaded to simulated pretightening force.
\end{abstract}

The results showed that the maximum stress $\left(\sigma_{\mathrm{vM}}\right)$ of each component occurred in the model with the longest implant length and smallest diameter (model 2).

Index Terms - Dental implant, implant diameter and length, finite element analysis, worst-case condition.

\section{INTRODUCTION}

With the significant technological development in the field of osseointegrated implants, dental implants have been used as a predictable therapy to restore missing teeth with high long-term implant survival rates associated with different prosthetic rehabilitations [1].

The most implemented material in manufacturing of dental implants is titanium of different grades of pureness, since this material promotes a stable and functional connection between the bone and the surface of the implant [2], [3].

Generally, the magnitude of stress created in the dental implant and its surrounding bone after the placement due to implant loading affects the success or failure of dental implant placement [4]. The weakness of osseointegration can cause failure after dental implant placement [5], such as bone loss, occurrence of micromovements and stress concentration at the surface of the bone and the implant. Hence, reducing stress in the bone-implant interface area

Manuscript received June 29, 2018; revised January 15, 2019.

Jinyang Zhang, Maisheng Li, and Xiao Zhang are with Guangzhou Janus Biotechnology Co.,Ltd., Guangzhou, China (e-mail: jy.zhang@gzjanus.com,ms.li@gzjanus.com,zx623@126.com).

Xianshuai Chen is with Foshan Angels Biotechnology Co., Ltd., Foshan, China (e-mail: xs.chen@gzjanus.com). can lead to reduction of osseointegration damage and improve dental implant stability inside the bone [6]. Moreover, osseointegration depends on many factors such as the type of loading applied, the length and diameter of the implant, its geometry and surface, the bone-implant surface, and the quality of surrounding bone [2].

Before clinical trials of the endosseous dental implant placement, the mechanical and dynamic fatigue behavior should be evaluated by the method of dynamic loading tests to identify its fatigue properties. Besides, it is very useful for comparing dental implants of different designs or sizes. However, this test simulates the functional loading of dental implant in combination with their premanufactured prosthetic components under "worst case" conditions. If a part of the endosseous dental implant is available in various dimensions or/and configurations, testing shall be carried out for the worst-case conditions within the recommended use. Besides, the choice of the worst-case testing must be justified. However, there are few articles focus on how to choose the worst-case conditions of dental implants with different size and configurations.

Thus, in this simulation study, the worst-case condition among the dental implant models was confirmed by assessing the effect of implant diameter (3.5, 4.3 and $5.0 \mathrm{~mm})$ and length (8 and $15 \mathrm{~mm}$ ) shifting on the stress of dental implant (implant body, angled abutment and central screw) using a three-dimensional finite element analysis.

\section{MATERIAL AND METHODS}

Six types of models (Model 1-6) of dental implants without threads were designed and established using CAD software SolidWorks. The implant body (pure titanium), abutment (titanium alloy) and central screw (titanium alloy) were assembled for generating 3-dimensional models of dental implant (Fig. 1a). In this study, the characteristics of the components used in each model were shown in Table1.

All implants were vertically embedded in acrylic resin, which was poured into cylindrical specimen holder in the central location. All angled abutments were fixed in the crown (Fig. 1b). In all models, the implant embedment depth was $8 \mathrm{~mm}$. The specimen holder clamped the implant body at a distance $3.0 \mathrm{~mm}$ apically from the nominal bone level. The distance of $3 \mathrm{~mm}$ was to provide a representative case with respect to bone loss. In this study, the screw threads of implant body and central screw were simplified to a smooth geometry, which was used in a primarily analysis for the study of stresses distribution that occur at the interface between implant body, abutment and central screw. 
TABLE I: CHARACTERISTICS OF THE COMPONENTS USED IN EACH MODEL

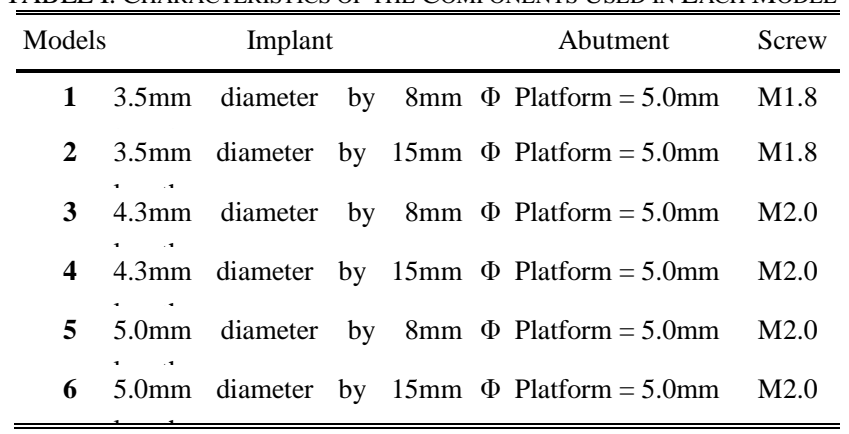
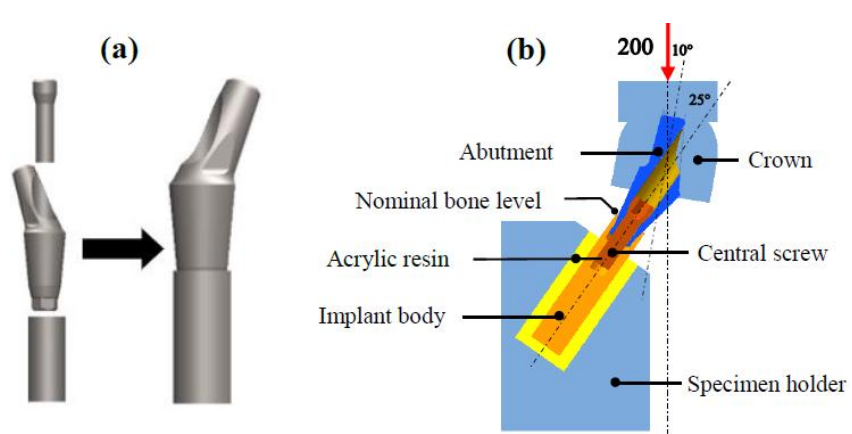

Fig. 1. (a) Assembled 3D models of dental implant including implant body, abutment and central screw, (b) Complete 3D model with a crown over the implant body which was embedded into acrylic resin.

In all the models, all materials were considered to be isotropic, homogeneous, and linearly elastic [7]. Poisson ratio (v) and Young modulus (E) were 0.33 and $105 \mathrm{GPa}$ for the implant body [8], 0.35 and 110GPa for the abutment and central screw [9], 0.3 and 220GPa for the crown [10], 0.3 and $1.37 \mathrm{GPa}$ for the acrylic resin [11], respectively.

The static analysis was performed by means of importing the selected 3-D models into the ANSYS finite element analysis software (ANSYS workbench). In this study, models were meshed with tetrahedral elements (Fig. 2). They were composed of 27976-39033 elements and 49047-67061 nodes.

To simulate the loads acting on the loading indenters, vertical shear-compression force of $200 \mathrm{~N}$ to the horizontal plane of crown was applied to the FE models (Fig. 1). Besides, a pre-torque of $30 \mathrm{~N} \cdot \mathrm{cm}$ on the central screw was loaded to simulated pretightening force. The interfaces between implant, abutment, central screw, resin, crown, and specimen holder were defined as "bonded" contacts. The translational and rotational degrees of freedom of the specimen holder were fully constrained so that the displacement of nodes in all directions was equal to zero.

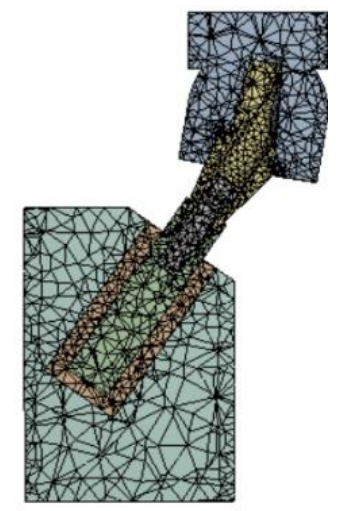

Fig. 2. The cross-sectional view of the meshed model 1.

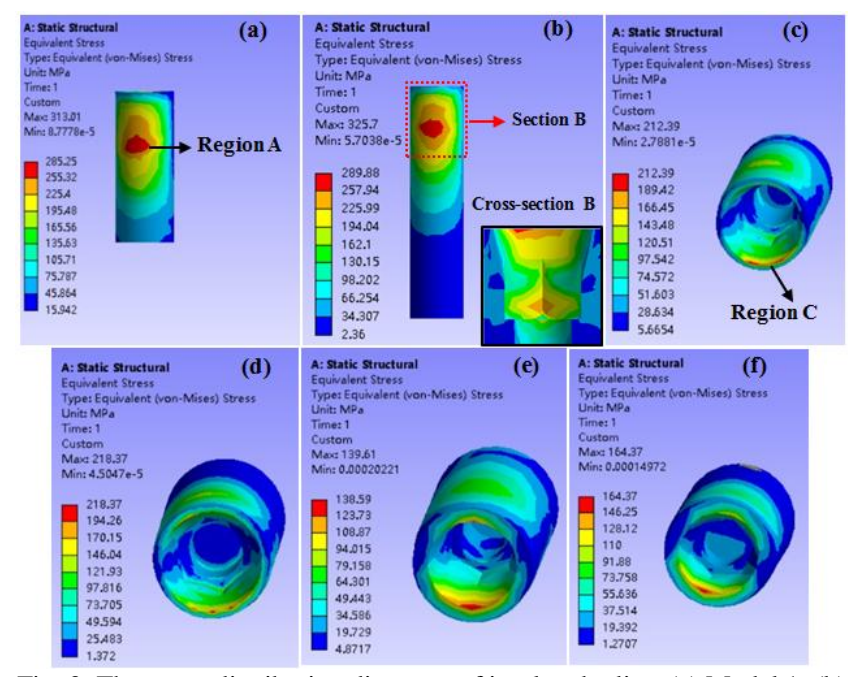

Fig. 3. The stress distribution diagrams of implant bodies: (a) Model 1; (b) Model 2; (c) Model 3; (d) Model 4; (e) Model 5; (f) Model 6.

\section{RESULTS}

\section{A. Stress Distribution}

\section{1) Implant body}

The stress distribution diagrams of implant bodies for all models were presented as follows (Fig. 3). It was clearly observed that the stress beard by the implant bodies in model 1-2 mainly centered at the neck area (Section B), where the upper region of the implant and specimen holder contacted (Fig. 3a and 3b). Moreover, there was also a higher level of stress was observed at the inner edge of implant top, as shown in the cross-sectional view of implant body in model 2 (Fig. 3b). The peak of $\sigma_{\mathrm{vM}}$ among model 4-6 was found occur at the inner edge of implant top (Fig. 3c, $3 \mathrm{~d}$ and $3 \mathrm{f}$ ), where the upper part of the implant and abutment contacted.

\section{2) Angled abutment}

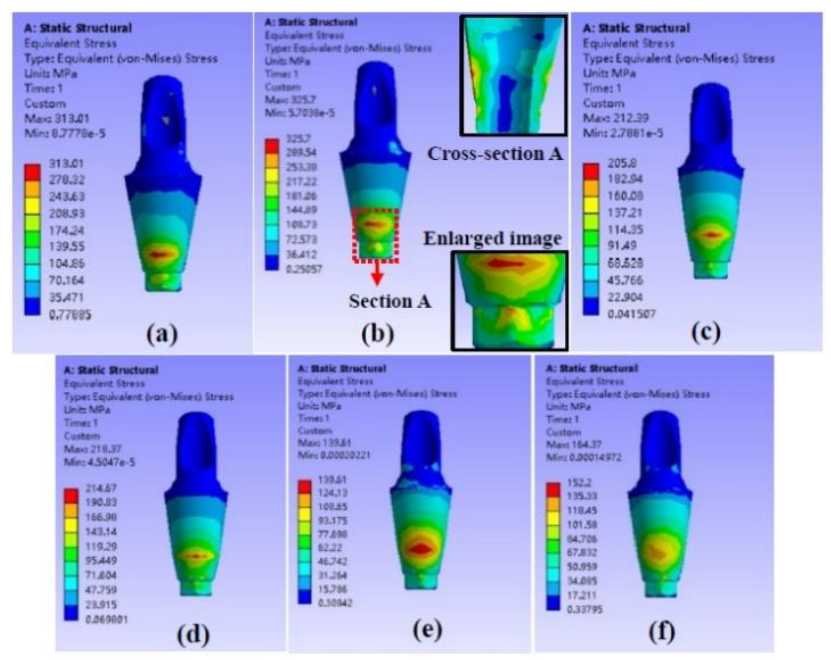

Fig. 4. The stress distribution diagrams of angled abutments with diameter of 5mm: (a) Model 1, (b) Model 2; (c) Model 3; (d) Model 4; (e) Model 5; (f) Model 6.

The stress distributions of angled abutments for all models illustrated that the peak of $\sigma_{\mathrm{vM}}$ tended to concentrate at the narrower region below the cervical collar of abutment (Fig. 4). Besides, in model 2, moderate to high stress was found at the top edge of the external hexagon of 
abutment as shown in the enlarged image (Fig. 2b). The cross-sectional view of angled abutment (Fig. 4b) in model 2 suggested that the peak of $\sigma_{\mathrm{vM}}$ mainly centered on the surface of the abutment. In all models, the value of $\sigma_{\mathrm{vM}}$ of the abutment in model 2 was the largest, which was $325.7 \mathrm{MPa}$. It was much higher than that the implant body (289.88MPa).

\section{3) Central Screw}

The peak of $\sigma_{\mathrm{vM}}$ in central screws of all models demonstrated that the maximum stress of central screw mainly located in the lower region in contact with implant (Fig. 5). The value of $\sigma_{\mathrm{vM}}$ in model 2 was the maximum among all the models, which was $233.87 \mathrm{MPa}$. It was much lower than that the implant body and angled abutment.

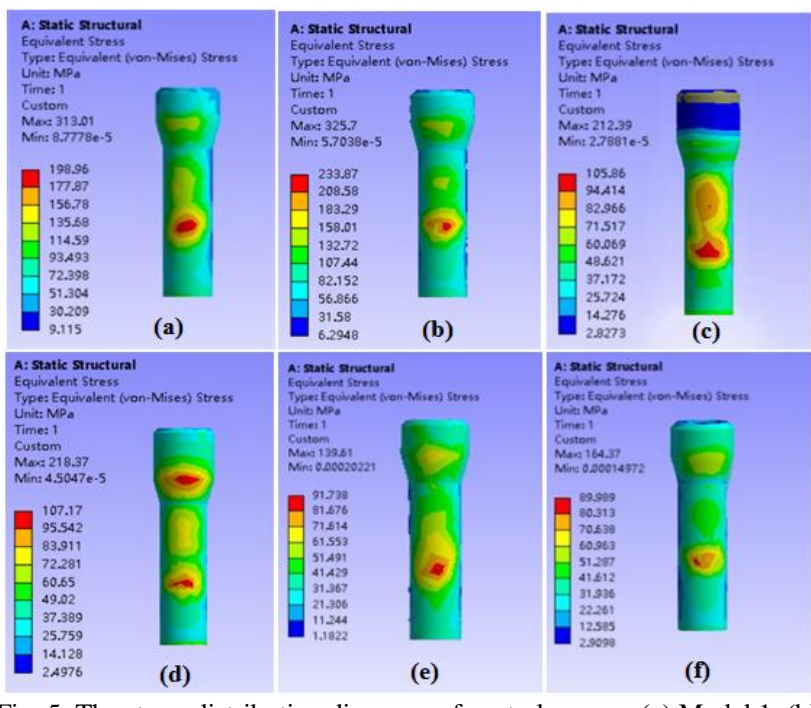

Fig. 5. The stress distribution diagrams of central screws: (a) Model 1, (b) Model 2; (c) Model 3; (d) Model 4; (e) Model 5; (f) Model 6.

\section{B. Influence of Implant Length and Diameter on the Stress of Dental Implant}

In all models, the maximum stress of each component indicated that the stress on implant body, abutment and central screw was strongly influenced by the implant length and diameter (Table II). It can be seen that the peak of $\sigma_{\mathrm{vM}}$ in model 2 was the maximum in all the models, which were $289.88 \mathrm{MPa}$ for the implant body, $325.70 \mathrm{MPa}$ for the abutment and 233.87MPa for the central screw (Table II).

TABLE II: Von-Mises EQuivalent STRESS $\left(\sigma_{v M}\right)$ OF EACH COMPONENT IN ALL MODELS

\begin{tabular}{cccc}
\hline \hline Models & \multicolumn{3}{c}{$\sigma_{\mathrm{vM}}(\mathrm{MPa})$} \\
\hline No. & Implant body & Abutment & Central screw \\
$\mathbf{1}$ & 285.25 & 313.01 & 198.96 \\
$\mathbf{2}$ & 289.88 & 325.70 & 233.87 \\
$\mathbf{3}$ & 212.39 & 205.80 & 105.86 \\
$\mathbf{4}$ & 218.37 & 214.67 & 107.17 \\
$\mathbf{5}$ & 138.59 & 139.61 & 91.73 \\
$\mathbf{6}$ & 164.37 & 152.20 & 89.98 \\
\hline \hline
\end{tabular}

\section{Influence of Implant Length}

The variation of the maximum stress as a function of the implant length showed that an increase in implant length lead to an increase in the value of $\sigma_{\mathrm{vM}}$ (Fig. 6). For the models with implant diameter of $3.5 \mathrm{~mm}$, the increment in the value of $\sigma_{v M}$ in implant body, abutment and central screw were $1.6 \%, 4.1 \%$ and $17.5 \%$ while the implant length increased from $8 \mathrm{~mm}$ to $15 \mathrm{~mm}$ (Fig. 6a). Similarly, the increment in the models with implant diameter of $4.3 \mathrm{~mm}$ were $2.8 \%, 4.3 \%$ and $1.2 \%$ (Fig. 6b), the increment in models with implant diameter of $5.0 \mathrm{~mm}$ were $18.6 \%, 9.0 \%$ and $-1.9 \%$ (Fig. 6c).

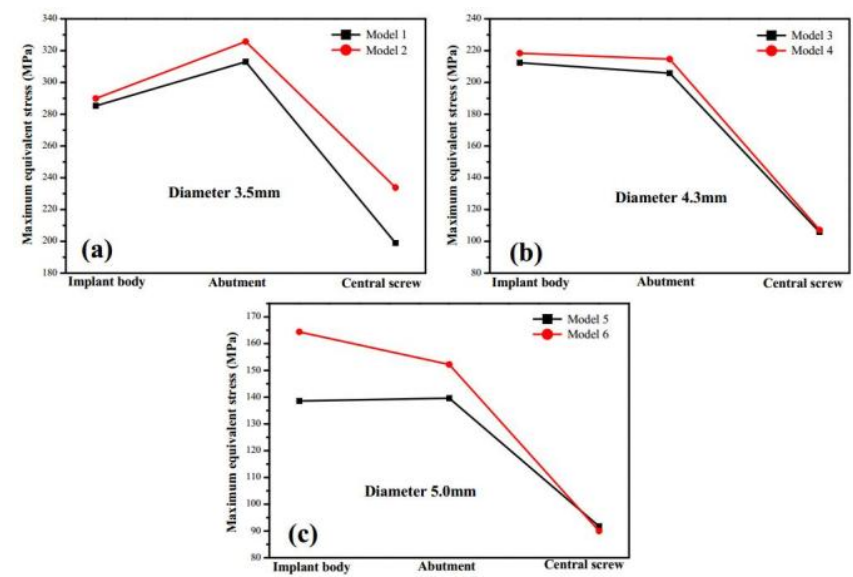

Fig. 6. Variation of the maximum stress $\left(\sigma_{\mathrm{vM}}\right)$ as a function of implant length when the implant diameter was (a) $3.5 \mathrm{~mm}$, (b) $4.3 \mathrm{~mm}$ and (c) $5.0 \mathrm{~mm}$ respectively.

\section{Influence of Implant Diameter}

A comparison with the maximum stress among components of dental implant with the same implant length but different diameters displayed distinct variances (Fig. 7). Keeping the implant length was $8 \mathrm{~mm}$ constant, the reductions in the value of $\sigma_{v M}$ in implant body, abutment and central screw were $25.5 \%, 34.2 \%$ and $46.8 \%$ when the implant diameter increased from $3.5 \mathrm{~mm}$ to $4.3 \mathrm{~mm}$. Stress continued to decrease for larger dimeter, the reductions were $34.7 \%, 32.2 \%$ and $13.3 \%$ accordingly when diameter increased to $5.0 \mathrm{~mm}$ (Fig. 7a).

Besides, by comparing the model 2 , model 4 and model 6 , the same regularity was found that the value of $\sigma_{v M}$ decreases as the increase of implant diameter when implant length was $15 \mathrm{~mm}$ (Fig. $7 \mathrm{~b}$ ).
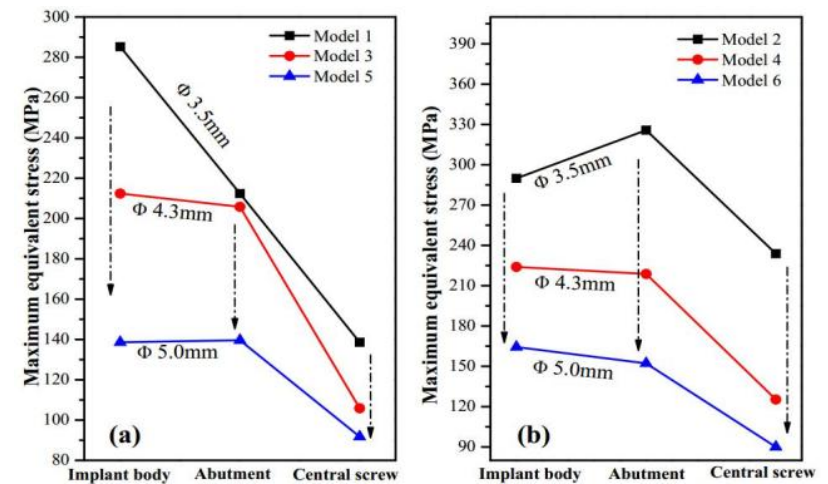

Fig. 7. Variation of the maximum stress $\left(\sigma_{\mathrm{vM}}\right)$ as a function of implant diameter when the implant length was (a) $8 \mathrm{~mm}$, and (b) $15 \mathrm{~mm}$ respectively.

\section{DISCUSSION}

Finite Element Analysis (FEA) is commonly used for 
stress analysis of nonrigid bodies to calculate strain, stress, deformation, etc. [12]. The results of the FEA computation depend on many individual factors, including material properties, boundary conditions, interface definition, and also on the overall approach to the model [13]. In this study, the implant body and central screw was simplified to cylinder, which was to establish the relative importance of implant length and diameter. Although screws are used more frequently in clinical applications, they were simulated as cylinder rather than screw-type implants in many $\mathrm{FE}$ analysis, for convenience of modeling [8], [14], [15]. Some studies have suggested that cylindrical implant models might be a good approximation of screw-type implants in further FE analysis [16].

However, this method cannot be applied to directly measure the stress or strain of implants system during the actual experimental test, the proposed model was only an approximation of the testing situation. Thus, in this simulation study, the explanation of the FEA results was done based on the previous published work [16]-[18].

For different sizes or design of endosseous dental implant, the worst case should be chosen for dynamic loading test before clinical trials. Considering the relevance of a fatigue resistant implant-abutment connection for the long-term clinical success, the combinations of 3 different implant diameters and 2 different implant lengths was evaluated using finite element method (FEM) in the present study. This research not only attempted to find the worst combination among the models of dental implant, but also provided some guidance on how to choose the worst case of dental implants with different implant diameters and lengths.

Primarily, the equivalent stress distribution of each component in all the models under loading $200 \mathrm{~N}$ in the vertical direction and loading a pre-torque of $30 \mathrm{~N} \cdot \mathrm{cm}$ on the central screw was investigated detailedly in this study. For implant body, the peak of $\sigma_{v M}$ mainly centered at the neck area in model 1-2 (Fig. 3a and 3b), which has been mentioned in previous published work [19]-[21]. Due to the upper part of the implant's connections in contact with specimen holder, a lever around the region A of implant body was generated under off-axis loading (Fig. 3a). As a result, a high stress concentration occurred in this region, which indicated a danger of overloading. However, the peak of $\sigma_{v M}$ in model 4-6 concentrated around the inner edge of implant top (Fig. 3b, 3c, 3d and 3f). In the contact area of abutment surface and inner edge of the implant top, a higher compressive stress in region $\mathrm{C}$ was formed when subjected to compressive forces (Fig. 3c). The above analysis can explain why two regions of strong stress concentration were observed in implant body. Furthermore, the peak of $\sigma_{v M}$ in abutment was located at the narrower region below the cervical collar (Fig. 4), which corresponded to the stress concentration zone (Region $\mathrm{C}$ ) in the form of interaction between abutment and implant body. In addition, the peak of $\sigma_{\mathrm{vM}}$ in the lower region of central screw was apparently observed (Fig. 5). In the case of contact between the upper part of the implant body and lower part of the central screw, a lever around the lower area of central screw was formed under off-axis loading, which caused great stress concentration.
On the other hand, the results of this study demonstrated that the implant diameters and lengths had an obvious effect on the stress distribution among components of dental implant. As the implant length increased, the value for $\sigma_{v M}$ exhibited a decreasing tend (Fig. 6a, 6b and 6c). Conversely, the value for $\sigma_{v M}$ declined with the increase of implant diameter (Fig. 7a and 7b). Besides, this trend was especially evident when evaluating implant diameters between 3.5 and $5.0 \mathrm{~mm}$. However, compared with the implant length, the effect of implant diameter on stress distribution of dental implant was more notable. It illustrated the fact that when using short implants in clinical situations, larger diameter implants might be preferred. Those findings were in agreement with other studies. Anitua E concluded that the effect of implant diameter on stress distribution in bone was more significant than the effect of the implant's length or its geometry [22]. Zhang included that increased orbital implant diameter better decreased the stress around the implant and the effect of implant length was less notable [23].

On the basis of these findings, it could be summarized that the peak of $\sigma_{v M}$ occurred in the model with the longest length and smallest diameter implant body.

The internal distribution of stresses acquired with FEA provides important data. Because of its ability to simulate complex geometries, material properties and various boundary conditions, it shows a great advantage in the application of dentistry, which is difficult to replicate in experiments [24]. In this study, only the effects of implant diameter and length on the stress distribution of dental implant were studied. However, the effect of other parameters, such as abutment's diameter, platform connection types (external hexagon or internal hexagon) and so on, need to be further study. Therefore, further virtual and mechanical trials with multiple combinations of implant-abutment connections are required for the selection guidelines to determination of worst-case conditions of dental implant with different designs or sizes.

\section{CONCLUSION}

In this paper, the effect of implant diameter and length on the stress distribution of dental implant was studied for determining the worst case among the six types of models. The results proved that the maximum stress occurred in the model with the longest implant length and smallest diameter. An increase in the implant length lead to an increase in the value of $\boldsymbol{\sigma}_{\mathbf{v M}}$. Conversely, the value of $\boldsymbol{\sigma}_{\mathbf{v M}}$ declined with the increase of implant diameter. Moreover, from a biomechanical perspective, the optimum choice was an implant with the maximum possible diameter and the shortest length allowed by the anatomy.

\section{ACKNOWLEDGEMENT}

This work was supported by 1) the Science and Technology Program of Nansha District, Guangzhou (Technology Development Program, No. 2017KF007); 2) the Science and Technology Program of Nansha District, Guangzhou (Application Technology Research, No. 2016GG020); 3) the Special Science and Technology 
Program of Guangdong, China (Application Technology Research, No. 2016B090925001).

\section{REFERENCES}

[1] D. Busenlechner, R. Haas, and G. Watzek, "Long-term implan success at the Academy for Oral Implantology: 8-year follow-up and risk factor analysis," Journal of Periodontal \& Implant Science, vol. 44, no. 3, pp. 102-108, 2014.

[2] P. Bicudo, J. Reis, and A. M. Deus, "Mechanical behaviour of dental implants," Procedia Structural Integrity, vol. 1, pp. 26-33, 2016.

[3] O. Babík, A. Czán, and J. Holubjak, "Identification of surface characteristics created by miniature machining of dental implants made of titanium based materials," Procedia Engineering, vol. 192, pp. 1016-1021, 2017.

[4] C. N. Elias, "Factors affecting the success of dental implants," IntechOpen: Implant Dentistry-A Rapidly Evolving Practice, vol. 27, pp. 93-101, 1990.

[5] H. S. Chang, Y. C. Chen, and Y. D. Hsieh, "Stress distribution of two commercial dental implant systems: A three-dimensional finite element analysis," Journal of Dental Sciences, vol. 8, no. 3, pp. 261-271, 2013.

[6] C. C. Lee, S. C. Lin, M. J. Kang, et al., "Effects of implant threads on the contact area and stress distribution of marginal bone," Journal of Dental Sciences, vol. 5, no. 3, pp. 156-165, 2010.

[7] S. Faegh and S. Müftü, "Load transfer along the bone-dental implant interface," Journal of Biomechanics, vol. 43, no. 9, pp. 1761-1770, 2010.

[8] G. Tepper, R. Hass, W. Zechner, W. Krach, and G. Watzek, "Tree-dimensional finite element analysis of implant stability in the atrophic posterior maxilla: A mathematical study of the sinus floor augmentation," Clinical Oral Implants Research, vol. 13, no. 6, pp. $657-665,2010$.

[9] L. Kong, K. Hu, D. Li, Y. Song, J. Yang, Z. Wu et al., "Evaluation of the cylinder implant thread height and width: A 3-dimensional finite element analysis," The International Journal of Oral \& Maxillofacial Implants, vol. 23, no. 1, pp. 65-74, 2008.

[10] E. Erkmen, G. Meric, A. Kurt, Y. Tunc, and A. Eser, "Biomechanical comparison of implant retained fixed partial dentures with fiber reinforced composite versus conventional metal frameworks: A 3D FEA study," Journal of the Mechanical Behavior of Biomedical Materials, vol. 4, no. 1, pp. 107-116, 2011.

[11] A. Lanza, R. Aversa, S. Rengo, D. Apicella, and A. Apicella, "3D FEA of cemented steel, glass and carbon posts in a maxillary incisor," Dental Materials, vol. 21, no. 8, pp. 709-715, 2005.

[12] J. Alsukhun, C. Lindqvist, M. Helenius, "Development of a three-dimensional finite element model of a human mandible containing endosseous dental implants. II. Variables affecting the predictive behavior of a finite element model of a human mandible,' Journal of Biomedical Materials Research Part A, vol. 80A, no. 1, pp. 247-256, 2010.

[13] S. Mohasseb and K. J. Bathe, Finite Element Procedures, 1996

[14] E. Kitamura, R. Stegaroiu, S. Nomura, and O. Miyakawa, "Influence of marginal bone resorption on stress around an implant - a three-dimensional finite element analysis," Journal of Oral Rehabilitation, vol. 32, no. 4, pp. 279-286, 2005.

[15] R. Stegaroiu, T. Sato, H. Kusakari, and O. Miyakawa, "Influence of restoration type on stress distribution in bone around implants: A three-dimensional finite element analysis," The International Journal of Oral \& Maxillofacial Implants, vol. 13, no. 1, pp. 82-90, 1998.

[16] N. Okumura, R. Stegaroiu, E. Kitamura et al., "Influence of maxillary cortical bone thickness, implant design and implant diameter on stress around implants: A three-dimensional finite element analysis," Journal of Prosthodontic Research, vol. 54, no. 3, pp. 133-142, 2010.

[17] L. Himmlová, T. Dostálová, A. Kácovský et al., "Influence of implant length and diameter on stress distribution: A finite element analysis," Journal of Prosthetic Dentistry, vol. 91, no. 1, pp. 20-25, 2004.

[18] Guan H. Application of the finite element method in dental implant research. Computer Methods in Biomechanics \& Biomedical Engineering, vol. 9, no. 4, pp. 257-270, 2006.
[19] Tada S, Stegaroiu R, Kitamura E, Miyakawa O, Kusakari H. Influence of implant design and bone quality on stress/strain distribution in bone around implants: a 3-dimentional finite element analysis. The International Journal of Oral \& Maxillofacial Implants, vol. 18 , no. 3 , pp. 357-368, 2003.

[20] Petrie CS, Williams JL. Comparative evaluation of implant designs: influence of diameter, length, and taper on strains in the alveolar crest. A three-dimensional finite-element analysis. Clinical Oral Implants Research, vol. 16, no. 3, pp. 486-494, 2005.

[21] H. J. Meijer, F. J. Starmans, W. H. Steen, and F. Bosman, "Loading conditions of endosseous implants in an edentulous human mandible: A three-dimensional, finite-element study," Journal of Oral Rehabilitation, vol. 23, no. 11, pp. 757-763, 2010.

[22] E. Anitua, R. Tapia, F. Luzuriaga, and G. Orive, "Influence of implant length, diameter, and geometry on stress distribution: A finite element analysis," The International Journal of Periodontics \& Restorative Dentistry, vol. 30, no. 1, pp. 89-95, 2010.

[23] Z. Xing, L. S. Chen, W. Peng, et al., "Influence of orbital implant length and diameter on stress distribution: A finite element analysis," Journal of Craniofacial Surgery, vol. 28, 2017.

[24] H. M. Huang, S. Y. Lee, C. Y. Yeh et al., "Resonance frequency assessment of dental implant stability with various bone qualities: A numerical approach," Clinical Oral Implants Research, vol. 13, no. 1, pp. 65-74, 2002.

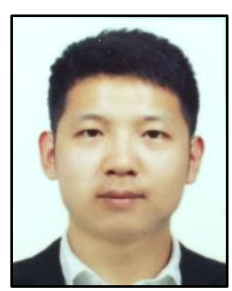

Jinyang Zhang is a $R \& D$ engineer. He has a master's and undergraduate degree in mechanical engineering. He lives in Guang Zhou, where his degrees were awarded. His current research interests are in the design and development of dental implants. During the graduate study in Guangdong University of Technology, he published two articles and a patent and won his Academic scholarship. Mr. Zhang is now engaged in research and development in the medical device industry.

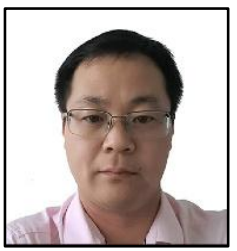

Maisheng $\mathbf{L i}$ is a mechanical design engineer. He has an undergraduate degree in material forming and control engineering at Jiamusi University. He lives in Foshan now. His current research interests are in the design and development of dental implants. He is now engaged in research and development in the medical device industry.

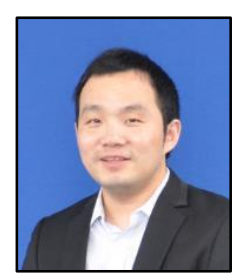

Xiao Zhang is a R\&D senior engineer. He has a master's degree in mechanical engineering. His current research interests are in the design and development of implantable medical devices and precision engineering. He has published 15 articles and 50 patents in this research fields.

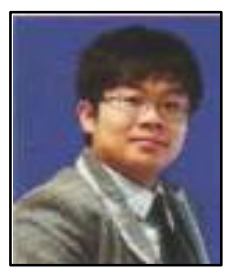

Xianshuai Chen is a managing editor of Chinese Journal of Tissue Engineering Research, a member of Youth Innovation Promotion Association CAS, Returned overseas talents. He received his Ph.D. degree from Chinese University of Hong Kong in 2009. His major research field is mechanical engineering, especially in precision engineering. He has published over 20 articles in foreign journals and SCI. conference, of which 20 have been indexed in the 